\title{
SYNTHESIS AND CHARACTERISTICS OF POLY(PARA-PHENYLENEVINYLENE)
}

\author{
Pham Thi Minh Chau \\ Institute of Geography- Vietnamese Academy of Science and Technology \\ Jung Il Jin \\ Center for Electro, Photo - Responsive Molecules, Korea University, \\ Seoul 136-701, Republic of Korea
}

Received 27 November 2006

\begin{abstract}
Poly(para-phenylenevinylene) [PPV] films and nanotubes were prepared via chemical vapor deposition polymerization (CVDP) by vapor phase pyrolysis of $\alpha, \alpha^{\prime}$ - dichloro p-xylene on the quartz plate, silicon wafers and $\mathrm{Al}_{2} \mathrm{O}_{3}$ membrane substrate with the pore size $\phi=100 \mathrm{~nm}$. We prepared graphitic carbon films and carbon nanotubes by carbonizing the poly(para-phenylene vinylene) $[\mathrm{PPV}]$ films and nano parterns under thermal treatment range from $500^{\circ} \mathrm{C}$ to $900^{\circ} \mathrm{C}$. When the PPV films on quartz plate were treated at $900^{\circ} \mathrm{C}$ highly oriented graphitic carbon films were obtained. The characterization of the PPV nanotube and nanotube carbon has been investigated by means of IR, SEM, AFM and Raman scattering study.
\end{abstract}

Keywords: Nanotube carbon, polymer nanotube

\section{INTRODUCTION}

Nowadays Poly(para-phenylenevinylene) PPV is still extensively studied due to its promising electrical, optical and electroluminescent properties [1,2]. The insoluble and infusible below the temperature range of decomposition cause to limit the synthesis, analysis and application of PPV. The polyreactions of PPV in solution result in oligomeric materials. The precursor route for making conjugated materials such as PPV allows to overcome this problem [3]. The chemical vapor deposition polymerization (CVDP) is a method, in which the surface of templates or substrates are utilized to accumulate polymer molecules. Jang et al. [4] prepared polypyrrol nanotubes by CVDP method on the inner surface of nano porous anodic aluminum oxide (AAO) membrane template that was presoaked in a $\mathrm{FeCl}_{3}$ aqueous solution. The deposited $\mathrm{FeCl}_{3}$ catalyzes the polymerization of the pyrrol monomers in the gas phase. The electrochemical polymerization is applicable also to the preparation of nanotubes of some of the polyconjugated polymer [5]. Nanotubes of soluble polymers simply can prepare by soaking the nano membranes or nano tubes in a polymer solution followed by the evaporation of the solvent. Polyaniline nanotubes and nanorods prepared by self-assembly method in the presence of an inorganic acid as dopant acid as $\mathrm{HCl}$ and $\mathrm{H}_{2} \mathrm{SO}_{4}$ [6]. Kyungkon $\mathrm{Kim}$ et al. prepared highly electrical graphitic carbon films and nano patterns by carbonizing poly(p-phenylenevinylene) films and nano patterns prepared on the silicon surface, nano porous alumina membrane by the chemical vapor deposition polymerization (CVDP) method of $\alpha, \alpha$-dichloro p-xylene [7, 8]. 
In the course of our studies, we used $\alpha, \alpha$-dichloro p-xylene for the preparation of PPV precursor and nano patterns on the substrate aluminum oxide $\left(\mathrm{Al}_{2} \mathrm{O}_{3}\right)$ membrane. We carbonized poly(p-phenylenevinylene) nano patterns inside the pores of $\mathrm{Al}_{2} \mathrm{O}_{3}$ membrane and on the surface of quartz plate. The characterizations of PPV nanotubes and carbon nanotubes were realized by IR, SEM, AFM, and Raman spectroscopy.

\section{EXPERIMENTS}

\subsection{Materials and measurements}

For synthesis of PPV via CVDP, we used commercally available nano porous alumina membranes (nominal pore diameter: $100 \mathrm{~nm}$, Whatman, England). The $\alpha, \alpha$-dichloro p-xylene (Aldrich Co, Milwaukee, U.S.A) was purified by recrystalization from benzene that was used as better monomers to produce PPVs with fewer chemical structural defects. The scanning electron microscopy (SEM, S-4300, Hidachi, Japan) and Atomic Force Microscope (AFM) were used to determine the morphology of nano patterns. The chemical structure of PPVs was observed through the infrared absorption spectra (Impact 400). Raman spectra were recorded by JobinYvon LabRam HR (high resolution), in which the set up of the $800 \mathrm{~nm}$ forcal length monochromator with $\mathrm{LN}_{2}$ cooled CCD multichanel detector. Ramanscattering experiments were performed at ambient conditions using a back scattering geometry for the laser exitation line: argon $514.5 \mathrm{~nm}$. The spectral resolution of the system was better than $2 \mathrm{~cm}^{-1}$.

\subsection{Synthesis}

The synthesis of PPV via CVD was obtained by vapor phase pyrolysis of $\alpha, \alpha^{\prime}$-dichloro pxylene. Figure 1 shows the pyrolysis apparature used for CVD process.

The CVD polymerization of the $\alpha, \alpha$-dichloro $p$-xylene monomer was conducted in a quartz tube consisting of vaporization, pyrolysis and deposition zone. The commercally available allumina porous filters (nominal diameter $100 \mathrm{~nm}$ Anodic Whatman, England) were utilized in the present investigation.

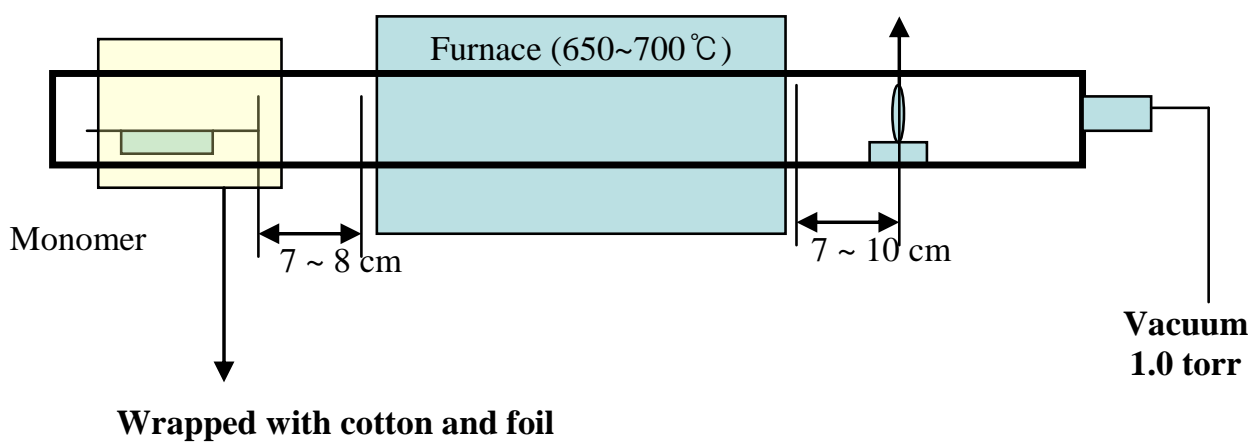

Fig. 1: The pyrolysis apparature for CVD process

A sample (40 - $60 \mathrm{mg}$ ) of purified from benzen $\alpha, \alpha$-dichloro p-xylene placed on tungsten boat was evaporized at $90-95^{\circ} \mathrm{C}$ in steady stream $8 \mathrm{ml} / \mathrm{min}$ of argon at the presure of 1.0 torr.

The evaporized monomer passes through the pyrolysis zone preheated to $650^{\circ} \mathrm{C}$. The precursor polymer was collected on the inner surface or inside the $\mathrm{Al}_{2} \mathrm{O}_{3}$ filter placed in the deposition zone. The precursor polymer nanotubes or nanofilms were thermally treated at $300^{\circ} \mathrm{C}$ for $12-15$ hours converting them into PPV. The PPV nanotubes and nanofilms were carbonized by heating 
at different temperatrures from $500-900^{\circ} \mathrm{C}$ for 1 hour under argon in the pyrolysis quartz tube to obtain the carbon tubes and carbon films.

\section{RESULTS AND DISCUSSION}

\subsection{Structure characterization}

Figure 2 shows the IR spectrum of the PPV. As indicated in the spectrum, $\mathrm{sp}^{3}$ saturated $\mathrm{C}-\mathrm{H}$ stretching absorption are observed at 2858 and $2945 \mathrm{~cm}^{-1}$. In addition, the absorption at 3024 $\mathrm{cm}^{-1}$ is belong to unsaturated $\mathrm{sp}^{2}=\mathrm{C}-\mathrm{H}$ stretching mode. The final PPV samples prepared by CVDP of $\alpha, \alpha$-dichloro p-xylene (bis 1,4-chlomethyl benzen) contain a low - level of the saturated 1,4-phenyleneethanediyl (PE) units in addition to the unsaturated 1,4phenylenevinylene (PV) units.

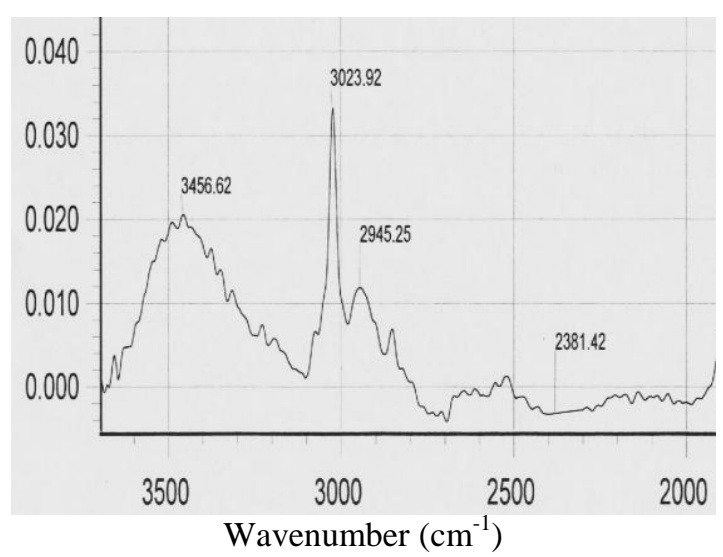

Fig. 2: IR spectrum of the PPV

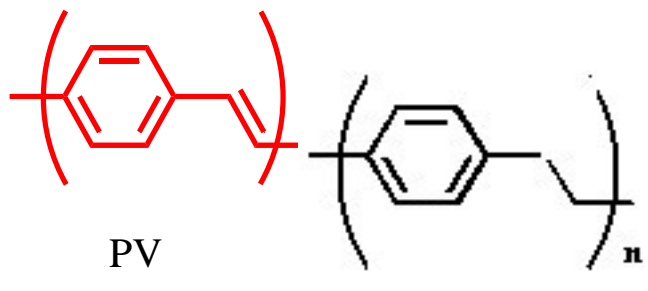

PE

Fig. 3: The formula of 1,4 phenylenevinylene $(P V)$ and 1,4-phenyleneethanediyl (PE) units

Figure 3 shows the formula of 1,4 phenylenevinylene (PV) and 1,4-phenyleneethanediyl (PE) units. This suggests that quinodimethane is formed together with quinodichloro upon pyrolyzing the monomer.

\subsection{Surface morphology}

The PPV nanotubes formed inside $\mathrm{Al}_{2} \mathrm{O}_{3}$ membrane by CVDP. Usually, the top surface of the membrane was covered by PPV formed during the CVDP process which was removed by $\mathrm{CF}_{4^{-}}$ plasma etching and 10\% hydroflourid solution. Figure 4 shows the scanning electron microscopy (SEM) images of PPV nanotubes. The PPV nano objects could be easily carbonized to the graphitic products by thermal treatment as above. The Atomic Force Microscopy (AFM) 
images of carbonized PPV nanotubes (CPNT) and carbon nanofilms were presented in Fig. 5. It is clear that the carbon particles on films and inside membrane were of the nanosize. The surface of the carbon films seems to be smooth and the cluster sizes were according to the results in Table 1.

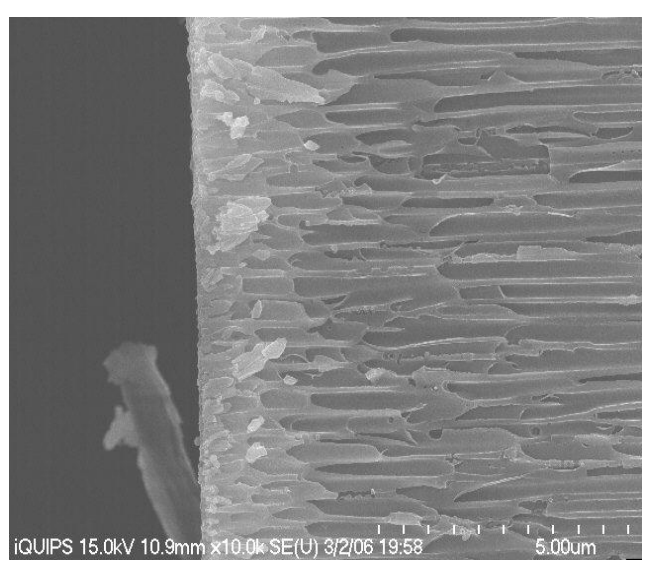

(a)

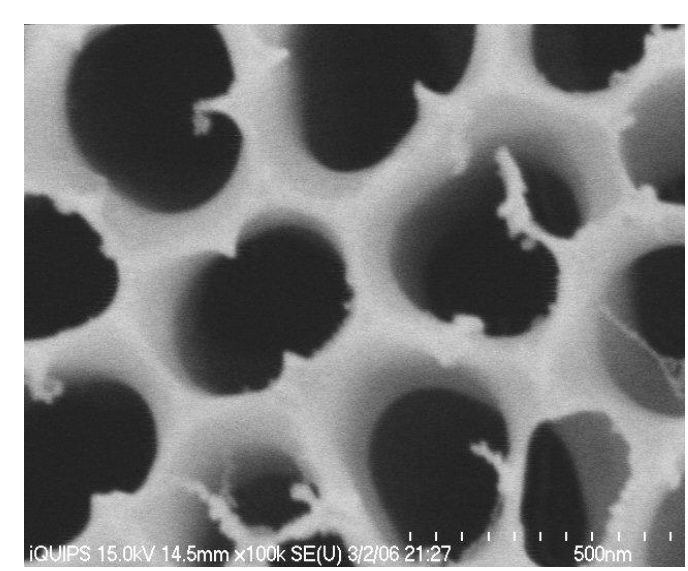

(b)

Fig. 4: (a) SEM images of nanotube PPV in an alumina filter membrane with nominal pore diameter of $100 \mathrm{~nm}$ from cross secsion. (b) Nanotube PPV inside Al2O3 membrane from top view

Table 1: Calculation results of graphitic cluster diameters

\begin{tabular}{ccccc}
\hline $\mathbf{N}^{\mathbf{0}}$ & Samples & Treatment temperature $\left({ }^{\mathbf{0}} \mathbf{C}\right)$ & $\mathbf{I}_{\mathbf{D}} / \mathbf{I}_{\mathbf{G}}$ & $\mathbf{L a}(\mathbf{n m})$ \\
\hline 1 & $\mathrm{NF}_{1}(\mathrm{ChAC} 500)$ & 500 & 0.5 & 9.0 \\
2 & $\mathrm{NF}_{2}(\mathrm{ChAC} \mathrm{700)}$ & 700 & 1.68 & 2.62 \\
3 & $\mathrm{NF}_{3}(\mathrm{ChAC}$ 900) & 900 & 1.87 & 2.35 \\
4 & $\mathrm{NTC}_{1}(\mathrm{ChAC} 17)$ & 700 & 2.35 & 1.87 \\
5 & $\mathrm{NTC}_{2}(\mathrm{ChAC} 18)$ & 900 & 2.31 & 1.90 \\
\hline
\end{tabular}

\subsection{Raman spectra}

Raman spectroscopy is a standard nondestructive tool for the characterization of crystalline, nano crystalline and amorphous carbons [8]. In the theoritical works [7, 9] the modes of Raman spectra in disordered and amorphous were given. However it is regreted that the experiments suggestions for nano object carbon obtained from PPV are not full. In order to describe in detail the Raman process of disordered carbons. We focuse on D and G Peaks, neglecting other features that sometimes present those at $1100-1400$ and $1500-1600 \mathrm{~cm}^{-1}$. Figure 6 shows the Raman spectra of the graphitized PPV films under $500^{\circ} \mathrm{C}(\mathrm{a}), 700^{\circ} \mathrm{C}(2), 900^{\circ} \mathrm{C}(1)$.

The Raman spectra of graphitized films (the curves in Fig. 6) display two peaks which correspond to the disordered carbon (D peaks) around $1350 \mathrm{~cm}^{-1}$ and a graphitic carbon (G peak) around $1580-1600 \mathrm{~cm}^{-1}$, usually assigned to zone center phonons of $\mathrm{A}_{1 \mathrm{~g}}$ symmetry and $\mathrm{K}$-point phonons of $\mathrm{E}_{2 \mathrm{~g}}$ symmetry, respectively. The $\mathrm{G}$ mode of graphite around $1600 \mathrm{~cm}^{-1}$ has $\mathrm{E}_{2 \mathrm{~g}}$ symmetry. This mode does not require the presence of six fold rings occured at $\mathrm{sp}^{2} \mathrm{sites}$, while it does in aromatic and olefinic molecules [9]. 


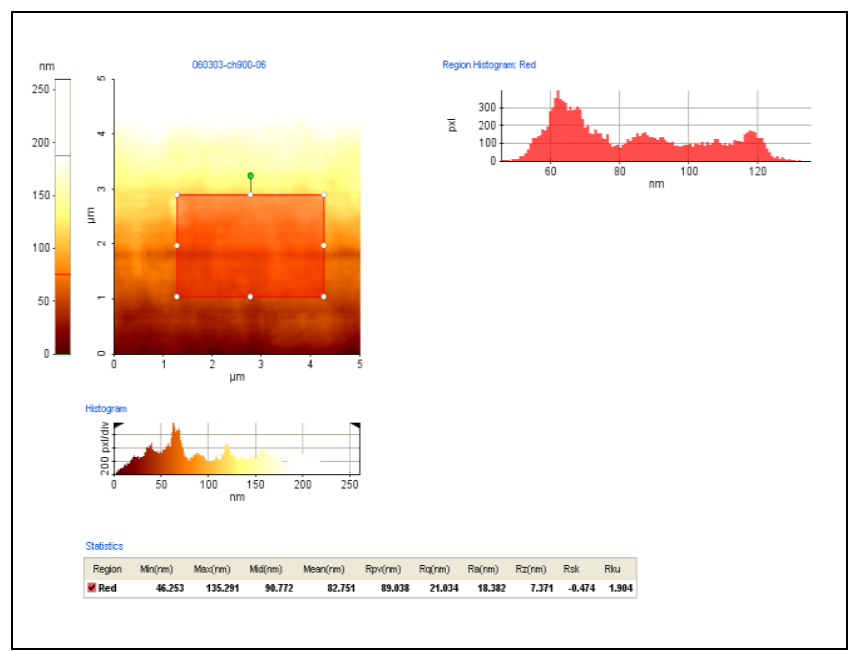

Fig. 5: AFM image of the nanocarbon film on quartz plate carbonazed PPV film at $500^{\circ} \mathrm{C}$ in 1 hour under Ar pressure

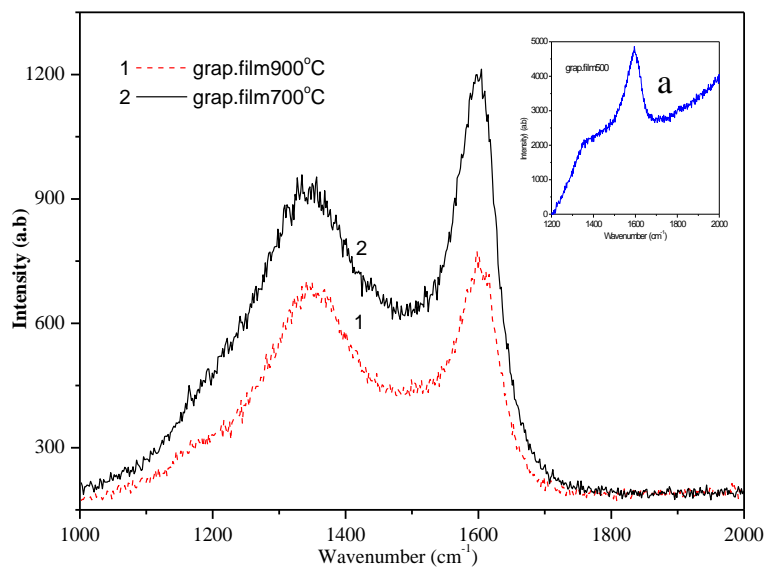

Fig. 6: Raman spectra of the graphitized PPV films under $900^{\circ} \mathrm{C}(1), 700^{\circ} \mathrm{C}(2), 500^{\circ} \mathrm{C}(\mathrm{a})$

The peak around $1350 \mathrm{~cm}^{-1}$ is very offen to called $\mathrm{D}$ peak which is a breathing mode of $\mathrm{A}_{1 \mathrm{~g}}$ symmetry involving phonons near the K-zone boundary. This mode is forbiden in perfect graphite and becomes active only in disordered graphites. The D mode is very dispersive and varies with photon excitation energy, even when the $G$ peak is not dispersive. The intensity of this mode is strickly connected to the presence of six fold aromatic rings.

The peak around $1600 \mathrm{~cm}^{-1}$ is the $\mathrm{G}$ mode of $\mathrm{E}_{2 \mathrm{~g}}$ symmetry [9] and it involves the in-plan bond stretching motion of $\mathrm{sp}^{2} \mathrm{C}$-atom [7]. Some authors used an alternative to a Gaussian fit line for the $\mathrm{G}$ peak and a Lorentzian for the D peak [9] or a double Gaussian fit line for the both peaks [7]. So, a Gaussian fit line has an asymmetric line shape and curve tails increasingly to lower frequencies for the lower coupling coefficient. The Gaussian + Lorentzian line is an excelent means to fit Raman spectra of all carbons from graphite to tetrahedral amorphous carbon (ta.C).

In general, the D peak position will decrease with increasing disorder with Gaussian + Lorentzian fit but will increase for the double - Gaussian fit [9]. When we utilized the Gaussian + Lorentzian fit, we obtained the $\mathrm{I}_{\mathrm{D}} / \mathrm{I}_{\mathrm{G}}$ ratio values of $0.5 ; 1.68 ; 1.86$; and 2.30 , respectively for 
the graphitic carbon films on quartz and nanotube inside $\mathrm{Al}_{2} \mathrm{O}_{3}$ membranes treated under 500; 700 , and $900^{\circ} \mathrm{C}$.

According to the Tuinstra and Koenig (TK) equation [10], the ratio of intensity to that of the G peak varied inversely with $\mathrm{La}$ (graphitic cluster diameter or in-plane correlation length):

$$
\mathrm{I}(\mathrm{D}) / \mathrm{I}(\mathrm{G})=\mathrm{C}(\lambda) / \mathrm{La}
$$

where $C(\lambda)$ is the scaling coefficent and depends on the wavelength of the source laser. The wavelength of the laser in our case was $514.5 \mathrm{~nm}$ and $\mathrm{C}(\lambda)$ is $44 \mathrm{~A}^{0}$. Table 1 tabulates the calculation results of $\mathrm{La}$ for the graphitic carbon films and nanotubes treated at different temperatures.

Ferrari A.C. and Robertson J. [9] present a three stage model relating the visible Raman parameters to the $\mathrm{sp}^{2}$ nano structure and the content of disordered carbons. The experimental visible Raman Spectra (on Fig. 7) of graphitic carbon films and carbon nanotubes were suitable to a phenomenological three stage model. The main effects in the evolution of the Raman spectrum of graphite to nano crystalline graphite are following:
a. The $\mathrm{G}$ peak moves from $1581 \mathrm{~cm}^{-1}$ a position close to $1600 \mathrm{~cm}^{-1}$
b. The $\mathrm{D}$ peak appears and $\mathrm{I}_{\mathrm{D}} / \mathrm{I}_{\mathrm{G}}$ increases following the equation (1)
c. There is no dispersion of the $\mathrm{G}$ mode

Table 2 tabulates the positions of the D-peak and the G-peak of the graphitic carbon films and carbon nanotubes.

Table 2: The positions of D-peak and G-peak of the nano objects

\begin{tabular}{ccccccc}
\hline $\mathbf{N}^{\mathbf{0}}$ & Samples & Treat. $\left({ }^{\mathbf{O}} \mathbf{C}\right)$ & $\mathbf{D}$ peak $(\mathbf{n m})$ & FWHM & G peak $(\mathbf{n m})$ & FWHM \\
\hline 1 & $\mathrm{NF}_{1}(\mathrm{ChAC} 500)$ & 500 & 1348 & 86.96 & 1600 & 60.87 \\
2 & $\mathrm{NF}_{2}(\mathrm{ChAC} 700)$ & 700 & 1336 & 172.18 & 1600 & 77.39 \\
3 & $\mathrm{NF}_{3}(\mathrm{ChAC} 900)$ & 900 & 1348 & 139.13 & 1604 & 78.26 \\
4 & $\mathrm{NTC}_{1}(\mathrm{ChAC} 17)$ & 700 & 1348 & 161.73 & 1604 & 80.86 \\
5 & $\mathrm{NTC}_{2}(\mathrm{ChAC} 18)$ & 900 & 1340 & 139.13 & 1602 & 78.26 \\
\hline
\end{tabular}

The physical properties of carbon materials strongly depend on the ratio of $\mathrm{sp}^{2}$ (graphite like) to $\mathrm{sp}^{3}$ (diamon like) bonds. The $\mathrm{G}$ peak displays around $1600 \mathrm{~cm}^{-1}$ in all the cases of our experiment graphitic carbon films and carbon nanotubes prepared by the CVDP method. The $\mathrm{I}_{\mathrm{D}} / \mathrm{I}_{\mathrm{G}}$ value is about 2,0 tells us that the obtained carbon films are the nanocrystalline graphite [7]. It is known that the $G$ peak moves from $1581 \mathrm{~cm}^{-1}$ to $1600 \mathrm{~cm}^{-1}$ when the disordered graphite becomes the nanocrystalline graphite.

\section{CONCLUSION}

We have successfully prepared the conjugated polymer (PPV), PPV nanofilms on quartz and inside the pore of $\mathrm{Al}_{2} \mathrm{O}_{3}$ membrane by the chemical vapor deposition polymerization of $\alpha, \alpha$ dichloro p-xylene and the graphitic carbon tubes, carbon films by carbonizing PPV. The increasing of the temperature in a graphitized process from 500 to $900^{\circ} \mathrm{C}$ leads to decrease the cluster diameter of nanocrystalline graphite films. 


\section{ACKNOWLEDGEMENT}

The authors gratefully acknowledge the support of Korea Science and Engineering Foundationn through the Center for Electro and Photo-Responsive Molecules of Korea University. This work was also partly supported by VN Basic Research Programs.

\section{REFERENCES}

1. Kathleen M. Vaeth and Klavs F. Jensen (1998), Macromolecules, vol. 31, pp. 6789-6793.

2. Kang, K.S., Kim, K.H., Kim, M.S., Park, K.T., Kim, K.M., Lee, T.H., Joo, J., Kim, K., Lee, D.W., and Jin, J.I. (2001), Current Applied Physics, vol. 1, pp. 443-446.

3. Kim, K.and Jin, J.-I. (2001), Nano Lett., vol. 1, p. 631.

4. Jang, J. and Oh, J.H. (2004), Chem. Commun., p. 882.

5. Fu, M., Chen, F., Zhang, J., and Shi, G.J. (2002), Mater. Chem., vol. 12, p. 2331.

6. Zhang, Z., Wei, Z., and War, M. (2002), Macromolecules, vol. 35, p. 5937.

7. Kyungkon Kim, Sae Chae Jeoung, Jin Woo Lee, Taeghwan Hyeon, and Jung-Il Jin (2003), Macromol. Symp., vol. 201, pp. 119-125.

8. Matthews, M.J., Pimenta, M.A., Dresselhaus, G., Dresselhaus, M.S., and Endo, M. (1999), Physical Review B, vol. 59(10), pp. 6585-6588.

9. Ferrari, A.C. and Robertson, J. (2000), Physical Revew B, vol. 61, p. 14095.

10. Tuinstra, F. and Koenig, J.L. (1970), The Journal of Chemical Physics, vol. 55(3), pp. $1126-1130$. 